\title{
Eudragit nanoparticles containing genistein: formulation, development, and bioavailability
} assessment

Jingling Tang ${ }^{2}$

$\mathrm{Na} \mathrm{Xu^{1,2 }}$

Hongyu Ji'

Hongmei Liu'

Zhiyong Wang'

Linhua $\mathrm{Wu}^{1,2}$

'Department of Pharmacy, the Second Affiliated Hospital of Harbin Medical University, Key Laboratory of College in Heilongjiang Province; ${ }^{2}$ Department of Pharmaceutics, School of Pharmacy, Harbin Medical University, Harbin, China
Correspondence: Linhua Wu

Department of Pharmacy, the Second

Affiliated Hospital of Harbin Medical

University, I57 Baojian Road,

Harbin, I50086, China

$\mathrm{Tel}+8645$ I 8660558 I

$\mathrm{Fax}+8645186665559$

Email yjhmu430@yahoo.com.cn
This article was published in the following Dove Press journal:

International Journal of Nanomedicine

18 October 2011

Number of times this article has been viewed

Background: Genistein, one of the major isoflavones, has received great attention as a phytoestrogen and potential cancer chemoprevention agent. However, the dissolution and bioavailability of genistein from solid oral preparations is low due to its poor water solubility.

Methods: In order to improve the oral bioavailability of genistein, genistein nanoparticles were prepared by the nanoprecipitation technique using Eudragit ${ }^{\circledR}$ E100 as carriers and an optimized formulation of mass ratio (genistein:Eudragit E100, 1:10). The mean particle size of genistein nanoparticles was approximately $120 \mathrm{~nm}$ when diluted 100 times with distilled water. The drugloaded nanoparticles were spherical on observation by transmission electric microscopy.

Results: Encapsulation efficiency and drug loading of the genistein nanoparticles were approximately $50.61 \%$ and $5.02 \%$, respectively. Release of drug from the genistein nanoparticles was two times greater than that from the conventional capsules. After administration of genistein suspension or genistein nanoparticles at a single dose of $100 \mathrm{mg} / \mathrm{kg}$ to fasted rats, the relative bioavailability of genistein from the nanoparticles compared with the reference suspension was $241.8 \%$.

Conclusion: These results suggested that a nanoparticle system is a potentially promising formulation for the efficient delivery of poorly water-soluble drugs by oral administration.

Keywords: bioavailability, dissolution, genistein, nanoparticles, nanoprecipitation technique

\section{Introduction}

Genistein $\left(5,7,4^{\prime}\right.$-triatomic isoflavone, see Figure 1$)$ is a primary active component of the soybean, kudzuvine root, scoparius, and other leguminous plants. As one of the most extensively studied isoflavones, it is generally recognized as a phytoestrogen. ${ }^{1}$ With its weak estrogenic and antiestrogenic properties, genistein is not only used as an antioxidant, ie, an inhibitor of protein tyrosine kinase or topoisomerase II, but is also used to induce cell differentiation. ${ }^{2}$ Asiatic women with a high intake of isoflavones from soy products have been shown to have a decreased risk of osteoporosis, cardiovascular disease, breast and uterine cancer, and climacteric syndrome. ${ }^{3,4}$ Although frequent reports demonstrate positive influences of isoflavones on human health, the clinical application of genistein is hindered by its disadvantages, including poor solubility in water and low serum levels after oral administration. ${ }^{5}$

Various drug delivery systems, including self-nanoemulsified systems, superparamagnetic systems, and chitosan microspheres, have been used to increase the dissolution and bioavailability of genistein. It is reported that the optimized formulation dissolved more than $90 \%$ of genistein over five minutes in a self-nanoemulsified drug delivery system. ${ }^{6}$ Genistein encapsulated in $\mathrm{Fe}_{3} \mathrm{O}_{4}$-carboxymethylated chitosan 


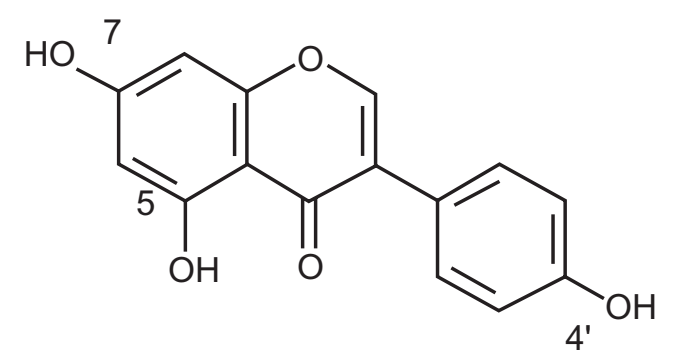

Figure I Structure of genistein.

nanoparticles shows greater water solubility than the free genistein. ${ }^{5}$

The Eudragit ${ }^{\circledR}$ E cationic copolymer has been widely used to improve the solubility of poorly water-soluble drugs. ${ }^{7}$ It has a basic site containing tertiary amine groups which are ionized in gastric fluid. ${ }^{8}$ Therefore, Eudragit $\mathrm{E}$ is easy to dissolve in the gastric environment. Eudragit $\mathrm{E}$ is one type of high polymer material, and is nontoxic, easily absorbed orally, and is widely used in coating and film forming. In recent years, it has been used to prepare microcapsules and nanoformulations to improve the solubility of poorly watersoluble drugs. It is reported that some new nanoparticle formulations of naringenin-Eudragit $E$ nanoparticles and quercetin-Eudragit $\mathrm{E}$ nanoparticles have been prepared for improving solubility., ${ }^{9,10}$

In this study, a drug delivery system for genistein was designed based on Eudragit E nanoparticles. It was expected that this system would improve the water solubility of the drug, and hence improve its bioavailability by oral administration. This novel nanoparticle system was characterized by its particle size, morphology, encapsulation efficiency, drug loading, and dissolution. Furthermore, the oral bioavailability of genistein nanoparticles was assessed and compared with that of a genistein suspension.

\section{Materials and methods Materials}

Genistein was purchased from Shanxi Huike Botanical Development Co Ltd (Xi'an, Shanxi, China). Standard phenacetin was supplied by the National Institute for Control of Pharmaceutical and Biological Products (Beijing, China). Sodium dodecyl sulfate was obtained from Tianjin Chemical Reagent Co Ltd (Tianjin, China). Pluronic $^{\circledR}$ F68 (Poloxamer 188) was kindly provided by BASF Corporation (Ludwigshafen, Germany). Filters $(0.22 \mu \mathrm{m}$ and $0.8 \mu \mathrm{m})$ were supplied by Millipore Corporation (Billerica, MA). Eudragit E100 was supplied by Röhm Pharma as a gift (Darmstadt, Germany). Sulfatase extracted from Helix pomatia was a product of Sigma-Aldrich Chemicals Co Ltd (St Louis, MO). All other reagents were of chromatographic grade. Distilled water, prepared from deionized water, was used throughout the study.

\section{Preparations of genistein nanoparticles}

The genistein nanoparticles were prepared by a nanoprecipitation method..$^{9,11,12}$ Genistein $5 \mathrm{mg}$ and Eudragit E $50 \mathrm{mg}$ were dissolved in $2 \mathrm{~mL}$ of ethanol. The internal organic phase solutions were slowly injected into $10 \mathrm{~mL}$ of the external aqueous solution (1\% Poloxamer 188 in water), and the mixtures were then stirred at $500 \mathrm{rpm}$ for 50 minutes. Internal organic phase solutions are always composed of solvents, making the drug and Eudragit soluble completely, and the external aqueous phase comprises aqueous solution, sometimes with surfactant in it. The surfactant can penetrate into the genistein nanoparticles during the nanoprecipitation process to form a stable nanoparticle delivery system. Ethanol was completely removed by rotary vacuum evaporation under a water bath at $32^{\circ} \mathrm{C}$. The nanoparticles formed were filtered using a $0.8 \mu \mathrm{m}$ filter and centrifuged at $11,000 \times \mathrm{g}$ for 60 minutes. The supernatant was separated and analyzed by high-pressure liquid chromatography (HPLC) for free drug content. The genistein nanoparticles formed were isolated, washed three times with distilled water, and freeze-dried. Lactose was chosen as a cryoprotectant, which had a good freezedrying effect. Blank nanoparticles were prepared following the above method without genistein. All the nanoparticles were prepared in triplicate.

\section{Particle size analysis}

The mean size of the genistein nanoparticles was determined using a LB-500 dynamic light scattering particle size analyzer (Horiba, Kyoto, Japan). Each sample was appropriately diluted 100 times with distilled water for analysis. The particle size analyzer could measure sizes ranging from $3 \mathrm{~nm}$ to $6000 \mathrm{~nm}$.

\section{Morphological analysis}

A drop of the fresh nanoparticle sample was placed onto a carbon-coated copper grid, forming a thin liquid film. The films on the grid were negatively stained by addition of a drop of $1 \%(\mathrm{w} / \mathrm{v})$ phosphotungstic acid. The excess staining solution was removed with filter paper, then air-dried. The stained films were observed on a Tecnai G2 transmission electron microscope (Philips, Eindhoven, The Netherlands) and photographed. 


\section{Encapsulation efficiency and drug loading}

The supernatant was separated by ultracentrifugation, ${ }^{13}$ and the encapsulation efficiency was determined by HPLC. ${ }^{14}$ The HPLC system consisted of a 600 pump (Waters, Milford, MA), a PDA-996 ultraviolet detector (Waters), and a 717 autosampler (Waters). A reversed-phase column (Diamonsil ${ }^{\mathrm{TM}} \mathrm{C}_{18}$, $250 \mathrm{~mm} \times 4.6 \mathrm{~mm}, 5 \mu \mathrm{m}$, Beijing, China) was utilized for drug separation at $25^{\circ} \mathrm{C}$. The mobile phase comprised a degassed mixture of methanol and water $(85: 15, \mathrm{v} / \mathrm{v})$. The flow rate of the mobile phase was maintained at $1.0 \mathrm{~mL} /$ minute and detection was performed at $261 \mathrm{~nm}$. A $10 \mu \mathrm{L}$ sample was injected into the HPLC column for analysis. The genistein concentration in the sample was determined using a calibration curve. The encapsulation efficiency and drug loading were determined using the following formulae:

Encapsulation efficiency $(\%)=$ encapsulated drug total drug $\times 100 \%$

Drug loading $(\%)=$ mass of genistein in nanoparticles /mass of nanoparticles recovered $\times 100 \%^{15}$

The method showed excellent reproducibility, with intraday and interday precision of less than $2 \%$ (relative standard deviation), as well as excellent accuracy between $91.7 \%$ and $100.0 \%$ for genistein. The lower limit of quantitation for genistein was $0.02 \mu \mathrm{g} / \mathrm{mL}$.

\section{Dissolution test}

The dissolution test was performed using a drug intelligence dissolution tester according to the second method of the Chinese Pharmacopoeia dissolution procedure. Briefly, lyophilized genistein nanoparticles in capsules and common capsules (drug filled in blank capsules) containing genistein approximately $1.6 \mathrm{mg}$ were put into different sinkers. Each sinker was loaded into $400 \mathrm{~mL}$ of phosphate buffer (pH 1.2) with $0.5 \%$ sodium dodecyl sulfate at $37^{\circ} \pm 0.5^{\circ} \mathrm{C}$ and a paddle speed of $100 \mathrm{rpm}$. Each sample $(4 \mathrm{~mL})$ was withdrawn at $5,10,20,30,45$, and 60 minutes, and an equal volume of temperature-equilibrated blank media was then added to the beaker. The samples were filtered using a $0.22 \mu \mathrm{m}$ filter, and $10 \mu \mathrm{L}$ was used for determination of genistein. The concentrations of drug were determined by HPLC analysis as already mentioned.

\section{Animals and drug administration}

The experimental protocols were approved by the animal care committee of Harbin Medical University, Heilongjiang, China. Twelve male Sprague-Dawley rats weighing 180-220 g were provided by the experimental animal center at our institution. They were housed in an air-conditioned room with temperature maintained at $25^{\circ} \mathrm{C} \pm 1{ }^{\circ} \mathrm{C}$ and humidity at $55 \% \pm 5 \%$ under a regular 12:12 hour light/dark cycle for one week prior to treatment. All animals were fed with standard rodent chow and water ad libitum. They were randomly divided into two groups, ie, one to receive genistein nanoparticles and the other to receive genistein suspension. The genistein suspension was prepared by suspending genistein in a $1 \%$ sodium carboxymethyl cellulose solution. All groups were fasted and given genistein suspension or genistein nanoparticles at a dose of $100 \mathrm{mg} / \mathrm{kg}$ by intragastric administration. Blood samples (approximately $0.25 \mathrm{~mL}$ ) were collected and put into heparinized tubes at hours $0.17,0.5,1,2,3,4,6,8$ and 12 after administration. Plasma samples were immediately separated by centrifugation at $3000 \times \mathrm{g}$ for 10 minutes and stored at $-20^{\circ} \mathrm{C}$ until analysis.

\section{Sample preparation}

The $100 \mu \mathrm{L}$ plasma samples were incubated with $100 \mathrm{U}$ of sulfatase at $37^{\circ} \mathrm{C}$ for eight hours, and phenacetin $10 \mu \mathrm{g} / \mathrm{mL}$ in mobile phase and $3 \mathrm{~mL}$ of methyl tert-butyl ether were then added to the samples in $5 \mathrm{~mL}$ Eppendorf tubes. After vortexmixing for three minutes and centrifugation at $3000 \times \mathrm{g}$ for 10 minutes, the supernatant was transferred to another tube and evaporated to dryness under a gentle stream of nitrogen at $40^{\circ} \mathrm{C}$. The residue was reconstituted with $100 \mu \mathrm{L}$ of mobile phase followed by vortex-mixing. A $20 \mu \mathrm{L}$ aliquot of the supernatant was injected into the HPLC system.

\section{HPLC assay}

The concentration of genistein was determined by HPLC using a 600 pump (Waters, Milford MA), a PDA-996 UV detector (Waters), and a 717 autosampler (Waters). A reversedphase column (Diamonsil ${ }^{\mathrm{TM}} \mathrm{C}_{18}, 250 \mathrm{~mm} \times 4.6 \mathrm{~mm}, 5 \mu \mathrm{m}$, Beijing, China) was utilized for drug separation at $30^{\circ} \mathrm{C}$. The mobile phase comprised a degassed mixture of methanol and water $(60: 40, \mathrm{v} / \mathrm{v})$. The flow rate of the mobile phase was maintained at $1.0 \mathrm{~mL} /$ minute. Detection was performed at $261 \mathrm{~nm}$. The method showed excellent reproducibility, with an intraday and interday precision of less than $12.1 \%$ (relative standard deviation), as well as an excellent accuracy of between $91.2 \%$ and $106.8 \%$ for genistein. The lower limit of quantitation for genistein was $0.15 \mu \mathrm{g} / \mathrm{mL}$. The extraction recovery of genistein and the internal standard was $55.55 \% \pm 6.65 \%$ and $52.88 \% \pm 2.96 \%$, respectively.

Genistein was stable in rat plasma samples after three freeze-thaw cycles, following benchtop storage at room 
temperature for 12 hours, and in reconstituted solution after being kept at room temperature for 12 hours. When stored at $-20^{\circ} \mathrm{C}$, the genistein added to the plasma samples was found to be stable for at least 30 days.

\section{Pharmacokinetic analysis}

The chromatographic data were automatically processed to obtain the peak-area ratio of compound to internal standard. The maximum plasma concentration $\left(C_{\max }\right)$ and the time to reach this peak concentration $\left(T_{\max }\right)$ were determined by visual inspection of the experimental data. The elimination rate constant $\left(K_{\mathrm{e}}\right)$ was calculated by applying the leastsquares regression technique to the data for the last three or four points of the plasma concentration-time curve, and the half-life $\left(t_{1 / 2}\right)$ of the drug was obtained by $0.693 / K_{\mathrm{e}}$. The area under the curve (AUC) was calculated by the trapezoid method. The relative bioavailability of genistein nanoparticles (test formulation) to the genistein suspension (reference formulation) was calculated using the following equation:

$$
\text { Relative BA }(\%)=\frac{\mathrm{AUC}_{\text {test }}}{\mathrm{AUC}_{\text {reference }}} \times \frac{\text { Dose }_{\text {reference }}}{\text { Dose }_{\text {test }}}
$$

The data were presented as the mean \pm standard error of the mean for the individual groups. The unpaired Student's $t$-test was used to determine any statistically significant differences. Differences were considered to be statistically significant at $P<0.05$.

\section{Results}

\section{Characterization of genistein nanoparticles}

Genistein nanoparticles prepared by the nanoprecipitation technique showed a blue opalescent and uniform appearance. The optimal encapsulation efficiency and drug loading of the genistein nanoparticle formulation were $50.61 \% \pm 0.41 \%$ and $5.02 \% \pm 0.04 \%$, respectively. The size of the genistein nanoparticles was approximately $120.0 \pm 9.25 \mathrm{~nm}$ when diluted 100 times with distilled water (Figure 2). The drug-loaded nanoparticles were small, spherical, and uniform, and there was no adhesion between particles seen on transmission electron microscopy (Figure 3).

\section{In vitro dissolution study}

Dissolution studies were performed for lyophilized genistein nanoparticles filled in capsules and for conventional genistein capsules. The dissolution rates of the drug from these

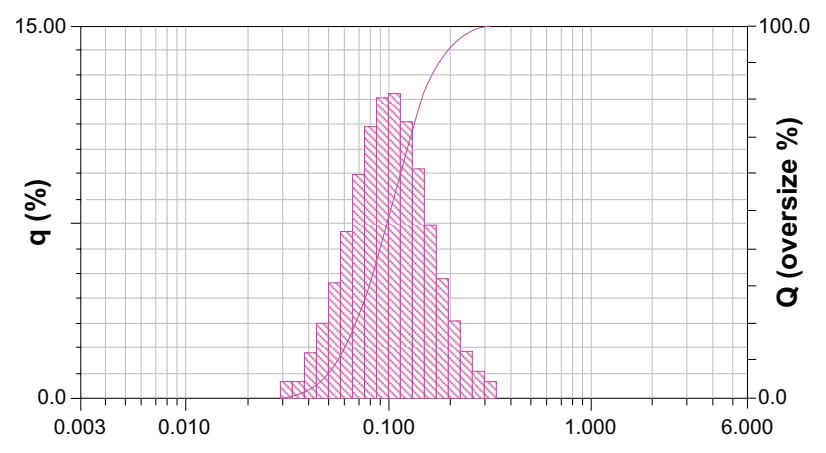

Figure 2 Size distribution of genistein nanoparticles.

preparations were evaluated in $\mathrm{pH} 1.2$ phosphate buffer with $0.5 \%$ sodium dodecyl sulfate. The release percentages of the drug from genistein nanoparticles were significantly higher than those from conventional genistein capsules (Figure 4). The release of genistein nanoparticles was more than $80 \%$ within 20 minutes. In contrast, less than $30 \%$ of genistein from the conventional genistein capsules dissolved within 20 minutes.

\section{Bioavailability studies}

The plasma profile of genistein in rats following oral administration of a single $100 \mathrm{mg} / \mathrm{kg}$ dose of genistein was investigated in the two groups. The plasma concentration profiles for genistein in the nanoparticles showed significantly greater improvement on oral absorption than did the genistein suspension (Figure 5). The main pharmacokinetic parameters for genistein in the two formulations are summarized in Table 1. The results show pronounced differences in the

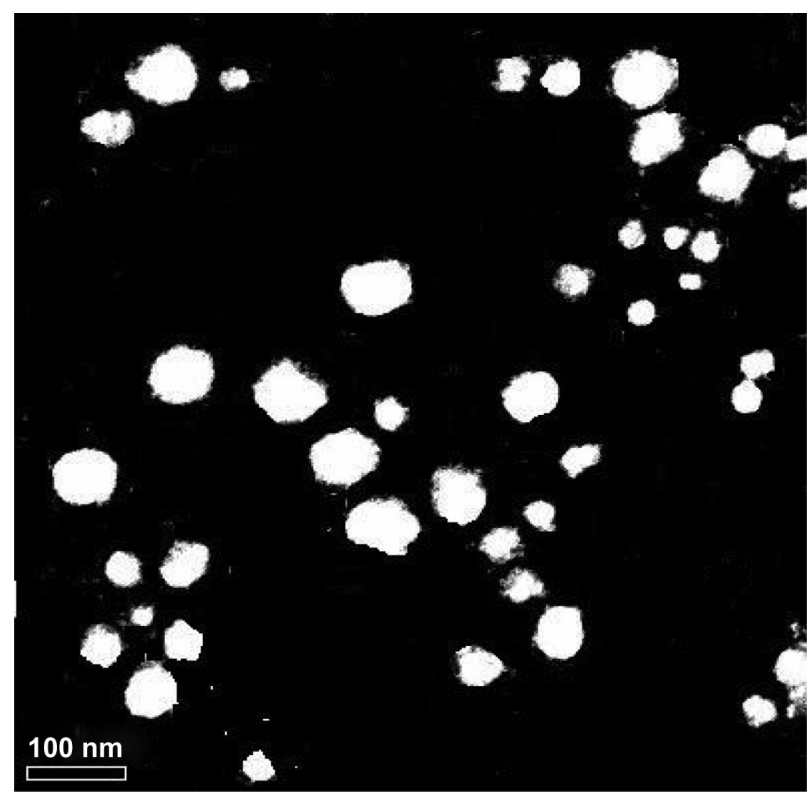

Figure 3 Transmission electron micrograph of genistein nanoparticles. 


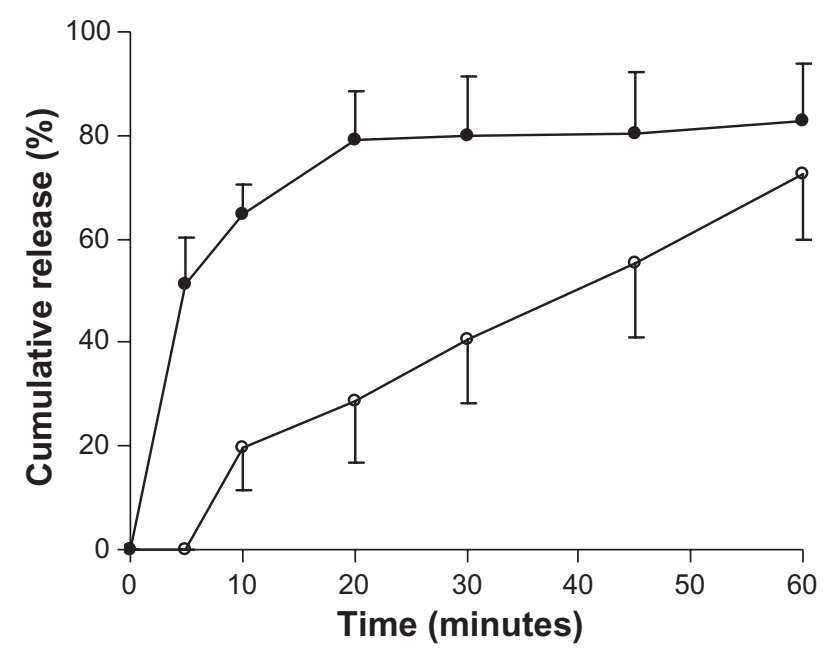

Figure 4 Dissolution profiles of genistein from genistein nanoparticles $(\bullet)$ and genistein capsules (O). Tests were conducted in $400 \mathrm{~mL}$ of $\mathrm{pH} 1.2$ phosphate buffer with $0.5 \%$ sodium dodecyl sulfate at $37^{\circ} \mathrm{C} \pm 0.5^{\circ} \mathrm{C}$ with a rotation speed of $100 \mathrm{rpm}$. Each value is the mean \pm standard deviation $(n=3)$.

pharmacokinetics of genistein following oral administration in rats. The difference in $C_{\max }$ value of $5.32 \pm 0.71 \mu \mathrm{g} / \mathrm{mL}$ in the formulation group and $2.17 \pm 0.87 \mu \mathrm{g} / \mathrm{mL}$ in the control group was statistically significant $(P<0.05)$. Marked differences were also observed for the AUC. The $\mathrm{AUC}_{0 \rightarrow 12}$ for the genistein suspension was $10.8 \mu \mathrm{g} / \mathrm{mL}$.hour and that for the genistein nanoparticles was $26.1 \mu \mathrm{g} / \mathrm{mL} \cdot$ hour $(P<0.05)$. The relative bioavailability of genistein from the nanoparticles compared with the suspension was $241.8 \%$, suggesting that a nanoparticle formulation could improve the oral absorption of genistein.

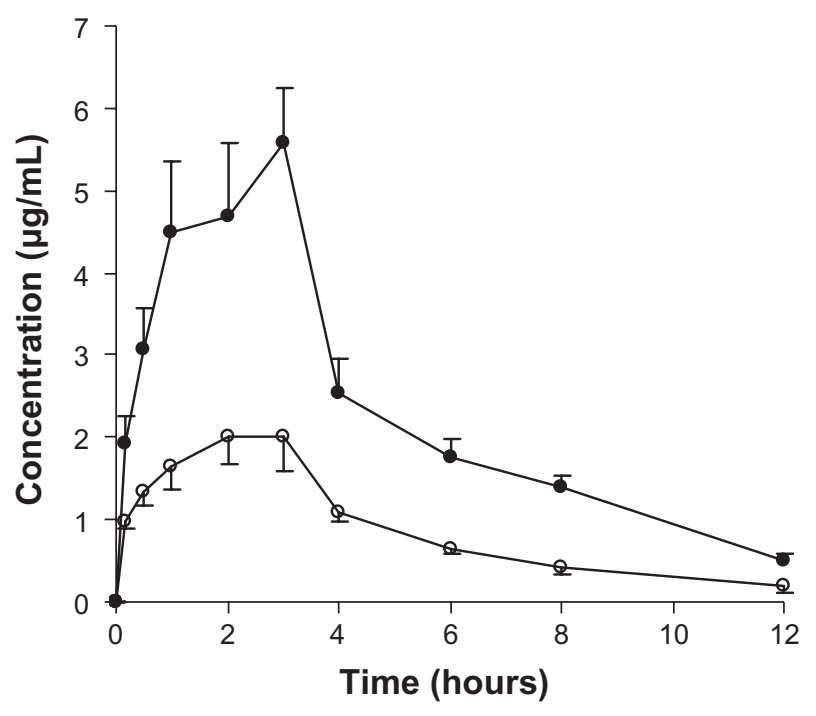

Figure 5 Genistein plasma concentration-time profiles of either genistein nanoparticles $(\bullet)$ or genistein suspension $(O)$ following oral administration of a single dose of $100 \mathrm{mg} / \mathrm{kg}$ genistein in rats. Each value is the mean \pm standard error $(n=6)$.
Table I Main pharmacokinetic parameters of genistein after a single $100 \mathrm{mg} / \mathrm{kg}$ oral dose of genistein in two groups, ie, a nanoparticle group and a suspension group

\begin{tabular}{lcl}
\hline Parameters & $\begin{array}{l}\text { Genistein } \\
\text { nanoparticles }\end{array}$ & Suspensions \\
\hline$C_{\text {max }}(\mu \mathrm{g} / \mathrm{mL})$ & $5.32 \pm 0.7 \mathrm{I}^{*}$ & $2.17 \pm 0.40$ \\
$T_{\text {max }}(\mathrm{h})$ & $2.50 \pm 0.34$ & $2.83 \pm 0.31$ \\
$K_{\mathrm{e}}\left(\mathrm{h}^{-1}\right)$ & $0.166 \pm 0.025$ & $0.198 \pm 0.030$ \\
$t_{1 / 2}(\mathrm{~h})$ & $4.60 \pm 0.59$ & $4.24 \pm 1.03$ \\
$\mathrm{AUC}_{0 \rightarrow 12 \mathrm{~h}}(\mu \mathrm{g} / \mathrm{mL} \cdot \mathrm{h})$ & $26.1 \pm 3.94^{*}$ & $10.8 \pm 0.87$ \\
Relative BA $(\%)$ & $241.8 \pm 32.79$ & 100 \\
\hline
\end{tabular}

Notes: Each value is the mean \pm standard error, $n=6, * p<0.05$.

Abbreviations: $C_{\max }$, peak plasma concentration; $T_{\text {max }}$, time to peak concentration; $t_{1 / 2}$, elimination half-life; AUC, area under the curve; $K_{e}$, elimination rate constant; Relative BA, relative bioavailability.

\section{Discussion}

In previous studies, various methods have been used to prepare nanoparticles, including a water-in oil-in-water $\left(\mathrm{w}_{1} / \mathrm{o} / \mathrm{w}_{2}\right)$ double emulsion method which is only used for hydrophilic drugs, and a film ultrasonic dispersion process which is complex, time-consuming, and difficult to control. Due to the limitations of these methods and the conditions of this experiment, the nanoprecipitation technique was used, based on its advantages of being relatively simple and rapid to perform. Moreover, it has been demonstrated in the literature that this nanoparticle delivery system can substantially transform the original physicochemical properties of drugs and greatly improve their bioavailability. ${ }^{16,17}$

For this experiment, a series of solvents, ie, ethanol, acetone, and dichloromethane, were used to investigate their influence on the preparation of genistein nanoparticles. Of these, the ethanol solvent was able to dissolve the drug and the polymer well and was quite clear, while acetone was more toxic than ethanol, and dichloromethane could not dissolve the drug or polymer completely. Therefore, ethanol was selected as the organic solvent for further study.

There were several reasons for enhancement of the drug dissolution rate. First, both genistein and Eudragit E are hydrophobic substances, which implies that a stronger affinity will be generated between them when blended with the organic ethanol solvent during the process of nanoparticle preparation. Second, the enhancement of drug dissolution could be attributed to the reduction of drug particle size, the enhanced hydrophilic properties of the drug when encapsulated in Eudragit E polymer, and enhanced wettability at the acidic $\mathrm{pH}$ provided by the dissolved Eudragit E. Finally, the hydrophilic and hydrophobic portion of Poloxamer 188 can penetrate into genistein nanoparticles during the nanoprecipitation process to form a stable nanoparticle 
delivery system. Similar observations have been reported elsewhere. ${ }^{9,18}$

Nanoparticle size is an important factor in gastrointestinal absorption, because only nanoparticles of appropriate size can be absorbed significantly. Several mechanisms for gastrointestinal absorption of nanoparticles have been reported, ie, cell bypass channel transport, intestinal epithelial cells cross-cell uptake, and collection of lymph nodes in the ileum (Peyer's patches) of microfold cells. ${ }^{19,20}$ Studies have documented that when rats are given PLGA particles sized $100 \mathrm{~nm}, 500 \mathrm{~nm}, 1 \mu \mathrm{m}$, and $10 \mu \mathrm{m}$ by oral administration, the amount of $100 \mathrm{~nm}$ particles captured by Peyer's patches is 10-250 times greater than that of the other particle sizes. ${ }^{21}$ Further studies have shown a range of particle sizes to be suitable for gastrointestinal absorption, ${ }^{22}$ and that nanoparticles of around $100 \mathrm{~nm}$ are absorbed several times more efficiently than those of larger size. ${ }^{21}$ The nanoparticles prepared in the present experiment were $120 \mathrm{~nm}$, and could be absorbed well by the gastrointestinal tract, and thus would play a key role in efficacy.

There has been some research reporting on different genistein doses being administered to rats. For example, genistein $40 \mathrm{mg} / \mathrm{kg}$ was orally administered in a pharmacokinetic rat model, ${ }^{23}$ and a dose of $480 \mathrm{mg} / \mathrm{kg}$ was used in another study to investigate toxicity. ${ }^{24}$ In the present study, an intermediate dose of $100 \mathrm{mg} / \mathrm{kg}$ was administered.

There has been a report of genistein being administered orally in healthy premenopausal women to investigate the pharmacokinetics of genistein. Pharmacokinetic analysis of the plasma concentration-time curves showed that the $t_{1 / 2}$ of genistein was 7.77 hours. ${ }^{25}$ Similar findings have been reported by other investigators. Pharmacokinetic analysis showed that the serum half-life of genistein was $4.53 \pm 1.40$ hours in rats. ${ }^{23}$ In the present study, the $t_{1 / 2}$ of genistein was $4.60 \pm 0.59$ hours.

The faster absorption of genistein nanoparticles than that of genistein alone is mainly due to the higher hydrophilicity and dispersion of the nanoparticles. There were differences in the plasma concentration-time profiles and pharmacokinetic parameters of genistein in individual rats after oral administration in the two groups. This can be attributed to the fact that the absorption of genistein and activity of sulfatase can be very different from each other.

Sulfatase is from Helix pomatia, and has deglucuronidation and desulfation activities. Glucoside conjugates and sulfate conjugates of genistein are the major naturally occurring isoflavones in soybean and soybean-based food products. After being absorbed, genistein exists in the systemic circulation in the form of multiple molecules, such as glucuronide and sulfate conjugates. ${ }^{26}$ These conjugates of genistein are converted to the aglycone form by sulfatase. Genistein glucuronides become active in vivo by hydrolysis, and only in this way can genistein have strong physiological functions. Sulfatase treatment is necessary because it plays an important role in the measurement of genistein concentration in plasma samples. A similar observation has been reported by other investigators. ${ }^{23,27}$

In conclusion, genistein, the principal soy isoflavone, is a molecule of great interest as a lead compound in anticancer drug design and an innovative chemotherapeutic agent. However, genistein is poorly soluble in water, and the dissolution and bioavailability of the drug from the solid oral preparations are different and not reproducible. In this study, the use of nanoparticles improved the dissolution of genistein. Furthermore, nanoparticles showed better oral absorption than the genistein suspension based on in vivo bioavailability studies. The relative bioavailability of genistein-loaded nanoparticles was $241.8 \%$ compared with the genistein suspension. In summary, Eudragit E nanoparticles offer a promising means to improve the solubility and bioavailability of genistein after oral administration.

\section{Disclosure}

The authors report no conflicts of interest in this work.

\section{References}

1. Rusin A, Krawczyk Z, Grynkiewicz G, Gogler A, Zawisza-Puchałka J, Szeja W. Synthetic derivatives of genistein, their properties and possible applications. Acta Biochim Pol. 2010;57:23-34.

2. Lamartiniere CA, Murrill WB, Manzolillo PA, et al. Genistein alters the ontogeny of mammary cancer development and protects against mammary cancer in rats. Proc Soc Exp Biol Med. 1998;217:358-364.

3. Usui T. Pharmaceutical prospects of phytoestrogens. Endocr J. 2006;53:7-20.

4. Cassidy A, Albertazzi P, Lise Nielsen I, et al. Critical review of health effects of soyabean phyto-oestrogens in post-menopausal women. Proc Nutr Soc. 2006;65:76-92.

5. Si HY, Li DP, Wang TM, et al. Improving the anti-tumor effect of genistein with a biocompatible superparamagnetic drug delivery system. J Nanosci Nanotechnol. 2010;10:2325-2331.

6. Zhu S, Hong M, Liu C, Pei Y. Application of Box-Behnken design in understanding the quality of genistein self-nanoemulsified drug delivery systems and optimizing its formulation. Pharm Dev Technol. 2009; 14:642-649.

7. Jung JY, Yoo SD, Lee SH, Kim KH, Yoon DS, Lee KH. Enhanced solubility and dissolution rate of itraconazole by a solid dispersion technique. Int J Pharm. 1999;187:209-218.

8. Wang SL, Lin SY, Chen TF, Cheng WT. Eudragit E accelerated the diketopiperazine formation of enalapril maleate determined by thermal FTIR microspectroscopic technique. Pharm Res. 2004;11:2127-2132.

9. Yen FL, Wu TH, Lin LT, Cham TM, Lin CC. Naringenin-loaded nanoparticles improve the physicochemical properties and the hepatoprotective effects of naringenin in orally-administered rats with $\mathrm{CCl}_{4}$-induced acute liver failure. Pharm Res. 2008;26:893-902. 
10. Wu TH, Yen FL, Lin LT, Tsai TR, Lin CC, Cham TM. Preparation, physicochemical characterization, and antioxidant effects of quercetin nanoparticles. Int J Pharm. 2008;346:160-168.

11. Ubrich N, Schmidt C, Bodmeier R, Hoffman M, Maincent P. Oral evaluation in rabbits of cyclosporin-loaded Eudragit RS or RL nanoparticles. Int J Pharm. 2005;288:169-175.

12. Zili Z, Sfar S, Fessi H. Preparation and characterization of poly-epsiloncaprolactone nanoparticles containing griseofulvin. Int $J$ Pharm. 2005;294:261-267.

13. Devarajan PV, Sonavane GS. Preparation and in vitro/in vivo evaluation of gliclazide loaded Eudragit nanoparticles as a sustained release carriers. Drug Dev Ind Pharm. 2007;33:101-111.

14. Sarmento B, Ribeiro A, Veiga F, Sampaio P, Neufeld R, Ferreira D. Alginate/chitosan nanoparticles are effective for oral insulin delivery. Pharm Res. 2007;24:2198-2206.

15. Govender T, Stolnik S, Garnett MC, Illum L, Davis SS. PLGA nanoparticles prepared by nanoprecipitation: drug loading and release studies of a water-soluble drug. J Control Rel. 1999;57:171-185.

16. Ratnam DV, Ankola DD, Bhardwaj V, Sahana DK, Kumar MN. Role of antioxidants in prophylaxis and therapy: A pharmaceutical perspective. J Control Release. 2006;113:189-207.

17. Dai J, Nagai T, Wang X, Zhang T, Meng M, Zhang Q. pH-sensitive nanoparticles for improving the oral bioavailability of cyclosporine A Int J Pharm. 2004;280:229-240.

18. Shah PP, Mashru RC, Rane YM, Thakkar A. Design and optimization of mefloquine hydrochloride microparticles for bitter taste masking. AAPS Pharm Sci Tech. 2008;9:377-389.
19. Hussain N, Jaitley V, Florence AT. Recent advances in the understanding of uptake of microparticulates across the gastrointestinal lymphatics. Adv Drug Deliv Rev. 2001;50:107-142.

20. Desai MP, Labhasetwar V, Amidon GL, Levy RJ. Gastrointestinal uptake of biodegradable microparticles: effect of particle size. Pharm Res. 1996;13:1838-1845.

21. Win KY, Feng SS. Effects of particle size and surface coating on cellular uptake of polymeric nanoparticles for oral delivery of anticancer drugs. Biomaterials. 2005;26:2713-2722.

22. Florence AT, Hillery AM, Hussain N, Jani PU. Nanoparticles as carriers for oral peptide absorption: studies on particle uptake and fate. $J$ Control Rel. 1995;36:39-46.

23. Kwon SH, Kang MJ, Huh JS, et al. Comparison of oral bioavailability of genistein and genistin in rats. Int J Pharm. 2007;337:148-154.

24. Xu JN, Wang QK, Cui T, Luo YS, Wang SW, Zheng YX. Study on reproductive toxicity of genistein I. General reproductive toxicity. Chinese Traditional and Herbal Drugs. 2003;34:830-832.

25. Setchell KD, Faughnan MS, Avades T, et al. Comparing the pharmacokinetics of daidzein and genistein with the use of 13C-labeled tracers in premenopausal women. Am J Clin Nutr. 2003;77:411-419.

26. Shelnutt SR, Cimino CO, Wiggins PA, Ronis MJ, Badger TM. Pharmacokinetics of the glucuronide and sulfate conjugates of genistein and daidzein in men and women after consumption of a soy beverage. Am J Clin Nutr. 2002;76:588-594.

27. Chen J, Lin H, Hu M. Absorption and metabolism of genistein and its five isoflavone analogs in the human intestinal Caco-2 model. Cancer Chemother Pharmacol. 2005;55:159-169.
International Journal of Nanomedicine

\section{Publish your work in this journal}

The International Journal of Nanomedicine is an international, peerreviewed journal focusing on the application of nanotechnology in diagnostics, therapeutics, and drug delivery systems throughou the biomedical field. This journal is indexed on PubMed Central,

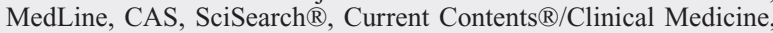

\section{Dovepress}

Journal Citation Reports/Science Edition, EMBase, Scopus and the Elsevier Bibliographic databases. The manuscript management system is completely online and includes a very quick and fair peer-review system, which is all easy to use. Visit http://www.dovepress.com/ testimonials.php to read real quotes from published authors. 